\title{
Nonlinear Dynamic Characteristics and Optimal Control of SMA Composite Wings Subjected to Stochastic Excitation
}

\author{
Zhi-Wen Zhu, ${ }^{1}$ Xin-Miao Li, ${ }^{1}$ and Jia $\mathrm{Xu}^{2}$ \\ ${ }^{1}$ Department of Mechanics, Tianjin University, Tianjin 300072, China \\ ${ }^{2}$ Tianjin Key Laboratory of Nonlinear Dynamics and Chaos Control, Tianjin 300072, China
}

Correspondence should be addressed to Jia Xu; xujia_ld@163.com

Received 31 May 2015; Revised 23 June 2015; Accepted 25 June 2015

Academic Editor: Nerio Tullini

Copyright (C) 2015 Zhi-Wen Zhu et al. This is an open access article distributed under the Creative Commons Attribution License, which permits unrestricted use, distribution, and reproduction in any medium, provided the original work is properly cited.

\begin{abstract}
A kind of high-aspect-ratio shape memory alloy (SMA) composite wing is proposed to reduce the wing's fluttering. The nonlinear dynamic characteristics and optimal control of the SMA composite wings subjected to in-plane stochastic excitation are investigated where the great bending under the flight loads is considered. The stochastic stability of the system is analyzed, and the system's response is obtained. The conditions of stochastic Hopf bifurcation are determined, and the probability density of the first-passage time is obtained. Finally, the optimal control strategy is proposed. Numerical simulation shows that the stability of the system varies with bifurcation parameters, and stochastic Hopf bifurcation appears in the process; the reliability of the system is improved through optimal control, and the first-passage time is delayed. Finally, the effects of the control strategy are proved by experiments. The results of this paper are helpful for engineering applications of SMA.
\end{abstract}

\section{Introduction}

High-aspect-ratio wings are applied in uninhabited air vehicles (UAV) widely. They have a great bending and torsion deformation under the flight loads, which induce the wing's fluttering. In this paper, a kind of shape memory alloy (SMA) composite wing is proposed to reduce the bending deformation. Shape memory alloy is a kind of smart material. It has many special properties, such as shape memory effect and damping characteristics. Moreover, SMA can be controlled by heating and cooling to achieve the structure deformation, which is helpful for reducing the wing's fluttering and ensuring the UAV's safety.

Several scholars have studied the application of shape memory materials in the wings. Yang et al. analyzed the characteristics of smart composite wing with SMA actuators [1]. Mirone designed adaptive airfoils and hingeless wings actuated by shape memory alloy wires [2]. Icardi and Ferrero studied an adaptive wing with shape memory alloy torsion actuators $[3,4]$. Barbarino et al. controlled the wing through an SMA-based device [5]. Grigorie and Botez proposed a new adaptive controller method for SMA hysteresis modelling of a morphing wing [6]. Kim and Cho designed a simple smart wing actuator using Ni-Ti SMA [7]. Barzegari et al. discussed the aeroelastic characteristics of cantilever wing with embedded shape memory alloys [8]. Kang et al. analyzed the morphing wing mechanism using an SMA wire actuator [9]. Colorado et al. researched the biomechanics of smart wings in a bat robot using SMA actuators [10]. Although much progress has been reported, the theoretical results of the dynamic characteristics of SMA composite wings are limited. For controlling the wing's fluttering efficiently, it is necessary to obtain the response and the dynamic characteristics of SMA composite wings.

A high-aspect-ratio SMA composite wing can be regarded as a cantilever SMA composite beam. There are also many researchers studying the dynamic characteristics of SMA beam. Lau discussed firstly the vibration characteristics of SMA beams with different boundary conditions [11]. Liew et al. studied the pseudoelastic behavior of an SMA beam by the element-free Galerkin method [12]. Zbiciak analyzed dynamic characteristics of pseudoelastic SMA beam [13]. Scarpa et al. developed spectral element formulation for SMA beams under random vibration excitation [14]. Hashemi and Khadem developed the dynamic model of SMA beam [15]. Collet et al. analyzed vibration behavior of SMA beam 


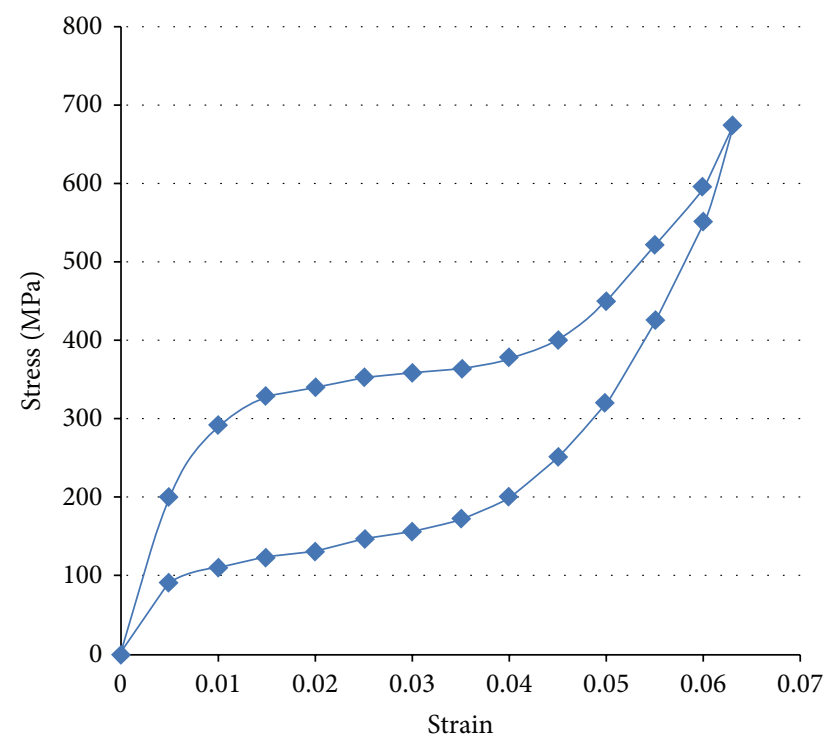

FIGURE 1: Strain-stress curves of Ti-Ni SMA.

under dynamical loading [16]. However, the airflow force is usually random to a low-flying UAV. It means that the UAV's high-aspect-ratio SMA composite wings are subjected to stochastic excitation. Although many achievements have been obtained, the dynamic characteristics of a cantilever SMA composite beam subjected to stochastic excitation are not abundant.

This paper aims to offer a kind of analysis method to the nonlinear dynamical characteristic of a cantilever SMA composite beam subjected to stochastic excitation. Nonlinear differential items are introduced to explain the hysteretic phenomenon of strain-stress curve of SMA, and the hysteretic nonlinear dynamic model of a cantilever SMA composite beam subjected to stochastic excitation is developed. The stochastic stability of the system is analyzed, and the system's response is obtained. The conditions of stochastic Hopf bifurcation are determined, and the probability density of the first-passage time is obtained. Finally, the optimal control strategy to improve the system's reliability is proposed.

\section{Modelling of SMA Material}

For obtaining the dynamic characteristics of a Ti-Ni SMA composite wing subjected to stochastic excitation, it is necessary to establish an accurate constitutive model of Ti-Ni SMA. The strain-stress curves of Ti-Ni SMA are presented in Figure 1, which are obtained by experiments. The SMA thin strip we used is supplied by Tianxin Company, Gansu Province, China. Its length is $12 \mathrm{~cm}$, its width is $0.35 \mathrm{~cm}$, and its thickness is $0.4 \mathrm{~mm}$. Evidently, hysteretic phenomena are present in Ti-Ni SMA strain-stress curves. Most of the current SMA models are shown as equations with piecewise functions or double integral functions owing to the hysteretic characteristics of Ti-Ni SMA [16-22]. As a result, it is hard to analyze the dynamic characteristics of SMA structure in theory. Usually, research results based on those models can only be obtained by numerical simulation or experiment [2327]. In [28], we proposed a Van de Pol hysteretic model about the relationship between the stress and the strain of SMA. In this paper, nonlinear differential items, which are developed from Van de Pol hysteretic model, are introduced to explain the hysteretic loop of Ti-Ni SMA strain-stress curves.

Van der Pol equation is a nonlinear equation. It can be shown as follows:

$$
\ddot{x}+x+\left(1-x^{2}\right) \dot{x}=0 .
$$

The item $\left(1-x^{2}\right) \dot{x}$ is called Van der Pol item. $\left(1-x^{2}\right) \dot{x}$ becomes $\pm\left(1-x^{2}\right)$ when $\dot{x}$ is \pm 1 . It means that the essences of Van der Pol item $\left(1-x^{2}\right) \dot{x}$ are two parabolic lines symmetrical about the original point $(0,0)$ when $\dot{x}$ is constant. Initial Van der Pol model can only be used in some basic parabolic hysteretic loop, where $\dot{x}>0$ means the loading process and $\dot{x}<0$ means the unloading process. For those hysteresis loops whose outset is $(0,0)$, Van der Pol item can be deformed as $\left(a x-b x^{2}\right) \dot{x}$, where $a$ and $b$ are the parameters which determine the width and height of the hysteretic loop [28].

However, the strain-stress curves of SMA are not pure parabolic lines. In this paper, a new differential item, which is developed from Van der Pol hysteretic model, is introduced to describe the strain-stress curves of SMA as follows:

$$
\begin{aligned}
\sigma & =\sigma_{1}+\sigma_{2} \\
& =a_{1} \varepsilon+a_{2} \varepsilon^{2}+a_{3} \varepsilon^{3}+\left(a_{4} \varepsilon+a_{5} \varepsilon^{2}+a_{6} \varepsilon^{3}+a_{7} \varepsilon^{4}\right) \dot{\varepsilon}
\end{aligned}
$$

where $\sigma$ is the stress, $\varepsilon$ is the strain, $a_{i}(i=1 \sim 7)$ are coefficients, $\sigma_{1}=a_{1} \varepsilon+a_{2} \varepsilon^{2}+a_{3} \varepsilon^{3}$ presents the skeleton curve of the hysteretic loop, and $\sigma_{2}=\left(a_{4} \varepsilon+a_{5} \varepsilon^{2}+a_{6} \varepsilon^{3}+a_{7} \varepsilon^{4}\right) \dot{\varepsilon}$ is the new nonlinear differential item, which describes the difference between the skeleton curve and the hysteretic loop. The item $f_{2}(\varepsilon)=\left(a_{4} \varepsilon+a_{5} \varepsilon^{2}+a_{6} \varepsilon^{3}+a_{7} \varepsilon^{4}\right) \dot{\varepsilon}$ is developed from the deformed Van der Pol item $\left(a x-b x^{2}\right) \dot{x} . a_{i}(i=1 \sim 7)$ are coefficients, which can be obtained by fitting method. From (2), we can see that the SMA material has nonlinear damping.

Equation (2) is the new model developed from the model we proposed in [28], and then we should test its accuracy. In this paper, the partial least-square regression software SIMCA-P is used to test the fitting effect. The procedure is as follows:

(1) Firstly, we obtained the stress-strain data of Ti-Ni SMA materials by experiments.

(2) Secondly, we choose the constructive model

$\sigma=a_{1} \varepsilon+a_{2} \varepsilon^{2}+a_{3} \varepsilon^{3}+\left(a_{4} \varepsilon+a_{5} \varepsilon^{2}+a_{6} \varepsilon^{3}+a_{7} \varepsilon^{4}\right) \dot{\varepsilon}$.

Please note that the coefficients $a_{i}$ are unknown at that time.

(3) Thirdly, we input the constructive model and the stress-strain data into the partial least-square regression software SIMCA-P; then the software will calculate the variable importance (VIP) and the coefficient values of each item. 


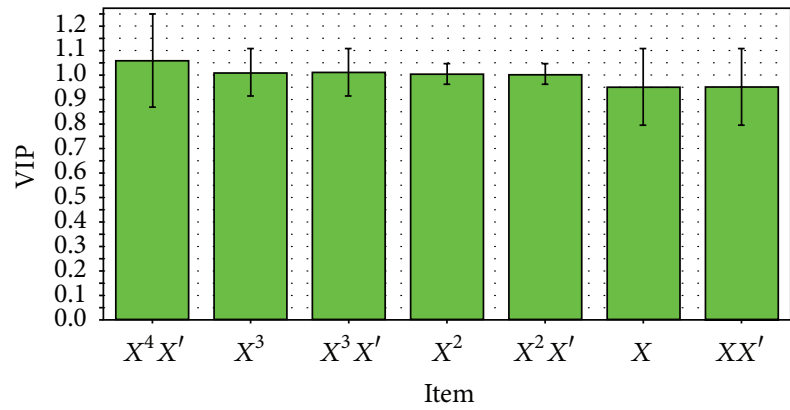

Figure 2: Variable importance of each item.

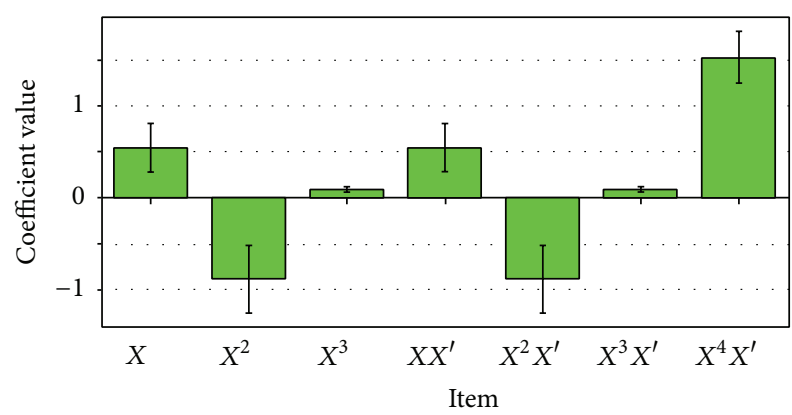

FIGURE 3: Coefficient values of each item.

(4) When the coefficient values of each item were given, the software can predict the stress-strain data according to the constructive model.

(5) Finally, the software can compare the stress-strain data from prediction and that of real stress-strain curves.

The analysis results of the principal component based on the experimental data are shown in Figure 2, and the coefficient values of each item are shown in Figure 3, where VIP is the variable importance. There is no item whose VIP is evidently little, which means that all items should be reserved in the constructive model.

The result of forecast test to (2) is shown in Figure 4, where the red lines present the experimental data and the black lines present the forecast values. Equation (2) can describe the experimental curves well.

\section{Modelling of SMA Composite Wing}

The research object is the Chinese "Wing Loong" UAV, which is shown in Figure 5. We can see that it has high-aspect-ratio wings. To control the wing's fluttering, we paste SMA thin plate on the inner surface of the wing. The SMA thin plate is made up of several SMA thin strips. The mechanical model of a high-aspect-ratio SMA composite wing is shown in Figure 6 . The composite wing can be regarded as a composite cantilever plate, where $a$ is the wing's length; $b$ is the wing's width, $h$ is the wing's thickness; $V=\bar{V}+B(t)$ is the relative velocity of airflow; $B(t)$ is Gauss white noise. $N=N_{0}+\bar{N} \varsigma(t)$ is the in-plane stochastic excitation, which is related with $V . \varsigma(t)$ is standard Gauss white noise whose mean is zero and intensity is $2 D, D>0$. In a high-aspect-ratio wing,

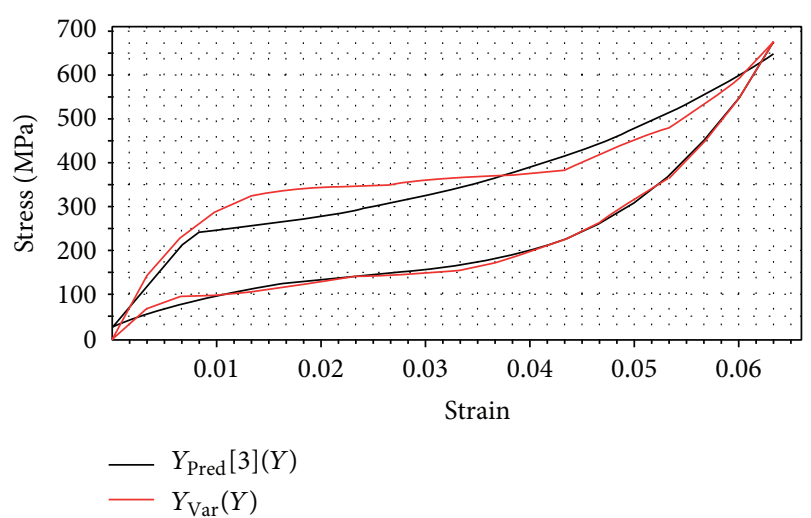

FIGURE 4: Results of the forecast test for the fitting effect of (2) on strain-stress data of SMA.

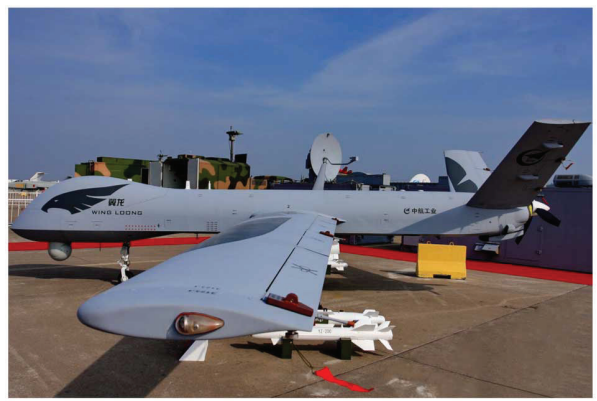

FIgURE 5: Chinese "Wing Loong” UAV.

there are great bending and torsion deformation. Usually, the dynamic model of a high-aspect-ratio wing can be shown as a two-degree-of-freedom (TDOF) system with two variables: vertical displacement and torsion angle. For a TDOF system subjected to stochastic excitation, it is hard to analyze its dynamic characteristics in theory. In this paper, the UAV is assumed as in level flight, and the torsion angle can be regarded as constant.

The boundary conditions of the composite wing can be written as follows:

$$
\begin{aligned}
x & =0: w=v=u=\phi_{y}=\phi_{x}=0, \\
x & =a: N_{x x}=N_{x y}=M_{x x} \\
& =M_{x y}-\bar{c}_{1} P_{x y}=\bar{Q}_{x}=0, \\
y & =0: N_{x y}=M_{y y}=M_{x y}-\bar{c}_{1} P_{x y} \\
& =\bar{Q}_{y}=0, \\
y & =b: N_{x y}=M_{y y}=M_{x y}-\bar{c}_{1} P_{x y} \\
& =\bar{Q}_{y}=0, \\
\left.\int_{-h / 2}^{h / 2} N_{y y}\right|_{y=0, \bar{b}} d z & = \pm \int_{-h / 2}^{h / 2}\left(F_{0}+F_{1} \cos \Omega_{1} t\right) d z,
\end{aligned}
$$




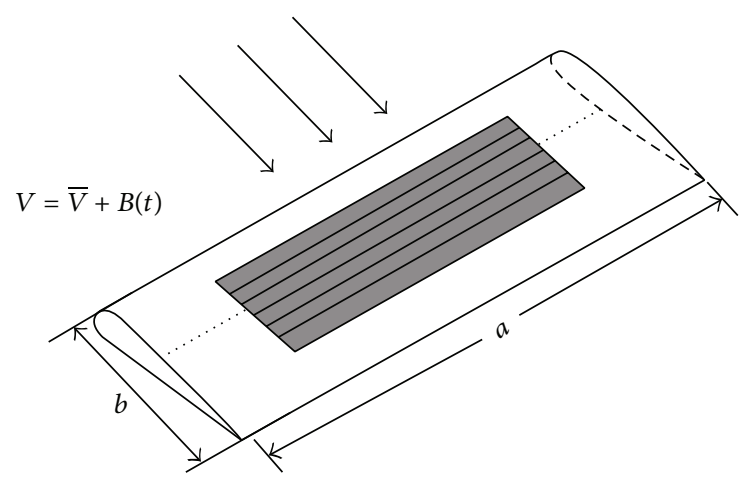

FIGURE 6: Mechanical model of a high-aspect-ratio SMA composite wing.

where

$$
\begin{aligned}
& \bar{Q}_{x}=Q_{x}+\frac{\partial M_{x y}}{\partial y}-\bar{c}_{2} R_{x}+\bar{c}_{1}\left(\frac{\partial P_{x x}}{\partial x}+\frac{\partial P_{x y}}{\partial y}\right), \\
& \bar{Q}_{y}=Q_{y}+\frac{\partial M_{x y}}{\partial x}-\bar{c}_{2} R_{y}+\bar{c}_{1}\left(\frac{\partial P_{y y}}{\partial y}+\frac{\partial P_{x y}}{\partial x}\right) .
\end{aligned}
$$
follows:

Thus, the vibration mode $u(x, y, t)$ can be assumed as

$$
u=u_{1} X_{1}(x) Y_{1}(y)+u_{2} X_{2}(x) Y_{2}(y)
$$

where

$$
\begin{aligned}
X_{i}(x)= & \sin \lambda_{i} x-\sinh \lambda_{i} x \\
& +\bar{\alpha}_{i}\left(\cosh \lambda_{i} x-\cos \lambda_{i} x\right), \\
Y_{i}(x)= & \sin \mu_{i} y-\sinh \mu_{i} y \\
& +\bar{\beta}_{i}\left(\cosh \mu_{i} y+\cos \mu_{i} y\right),
\end{aligned}
$$

$\cos \lambda_{i} \bar{a} \cosh \lambda_{i} \bar{a}+1=0$,

$\cos \mu_{i} \bar{b} \cosh \mu_{i} \bar{b}+1=0$,

$$
\begin{aligned}
& \bar{\alpha}_{i}=\frac{\sinh \lambda_{i} \bar{a}+\sin \lambda_{i} \bar{a}}{\cosh \lambda_{i} \bar{a}+\cos \lambda_{i} \bar{a}}, \\
& \bar{\beta}_{i}=\frac{\sinh \mu_{i} \bar{b}-\sin \mu_{i} \bar{b}}{\cosh \mu_{i} \bar{b}-\cos \mu_{i} \bar{b}} .
\end{aligned}
$$

Considering the complex characteristics of composite materials, we introduce Hamilton's principle to dynamic modeling of the system. The Hamilton function can be presented as

$$
S=T_{1}+T_{2}-U_{1}-U_{2}+W,
$$

where $T_{1}$ is kinetic energy of the wing, $T_{1}=$ $(1 / 2) \int_{0}^{L} \rho_{1} A_{1}(\partial u / \partial t)^{2} d x ; T_{2}$ is the kinetic energy of the SMA plate, $T_{2}=(1 / 2) \int_{0}^{L} \rho_{2} A_{2}(\partial u / \partial t)^{2} d x ; U_{1}$ is the potential energy of the wing, $U_{1}=\int_{0}^{L}\left(E_{1} I_{2} / 2\right)\left(\partial^{2} u / \partial x^{2}\right)^{2} d x+$
$\left(E_{1} A_{1} / 8 L\right)\left[\int_{0}^{L}(\partial u / \partial x)^{2} d x\right]^{2} ; U_{2}$ is the potential energy of the SMA plate, $U_{2}=(1 / 2) E_{2} A_{2} \int_{0}^{L}(\partial u / \partial x)^{2} d x ; W$ is the power of the external force, $\delta W=\int_{0}^{L} \delta u N d x ; \rho_{j}(j=1,2)$ is the density of the materials (1: wing, 2 : SMA plate); $E_{j}(j=1,2)$ is the elastic modulus; $A_{j}(j=1,2)$ is the area of cross sections.

According to the Hamilton principle,

$$
\delta S=\int_{t_{1}}^{t_{2}} \delta\left(T_{1}+T_{2}-U_{1}-U_{2}+W\right) d t=0 .
$$

Thus, the nonlinear dynamic model of the SMA composite wing can be shown as follows:

$$
\begin{gathered}
m \frac{\partial^{2} u}{\partial t^{2}}+\left[c+\int_{0}^{L}\left(a_{4} u+a_{5} u^{2}+a_{6} u^{3}+a_{7} u^{4}\right) d x\right] \frac{\partial u}{\partial t} \\
-a_{1} \frac{\partial^{2} u}{\partial x^{2}}-a_{2} \frac{\partial^{4} u}{\partial x^{4}}-a_{3} \frac{\partial^{2} u}{\partial x^{2}} \int_{0}^{L}\left(\frac{\partial u}{\partial x}\right)^{2} d x \\
=\frac{1}{6}\left(E A_{2} \frac{\partial^{2} u_{1}}{\partial x^{2}}+\rho_{2} A_{2} \frac{\partial^{2} u_{1}}{\partial t^{2}}\right) .
\end{gathered}
$$

According to (6), the dynamic equation of the system's response can be solved from (10) by the Galerkin method as follows:

$$
\begin{aligned}
\ddot{u}_{1} & +2 \eta_{1} \dot{u}_{1}+c_{1} u_{1}+c_{2} u_{1}^{3}+\gamma u_{1}^{2} u_{2}+\left(c_{3} u_{1}^{2}+c_{4} u_{1}^{4}\right) \dot{u}_{1} \\
& =f_{1} u_{1} \varsigma(t), \\
\ddot{u}_{2} & +2 \eta_{2} \dot{u}_{2}+e_{1} u_{2}+e_{2} u_{2}^{3}+\gamma u_{1}^{2} u_{2}+\left(e_{3} u_{2}^{2}+e_{4} u_{2}^{4}\right) \dot{u}_{2} \\
& =f_{2} u_{2} \varsigma(t) .
\end{aligned}
$$

When the wing's length is more than its width, which means $a \gg b$, the coupling coefficient $\gamma$ is close to zero, and the composite wing can be simplified as a composite beam. In this case, the dynamic equation of system response can be shown as follows:

$$
\ddot{u}_{1}+c_{1} u_{1}+c_{2} u_{1}^{3}+\left(2 \eta_{1}+c_{3} u_{1}^{2}+c_{4} u_{1}^{4}\right) \dot{u}_{1}=f_{1} u_{1} \varsigma(t),
$$

where $\eta_{1}$ is the damping coefficient and

$$
\begin{aligned}
& c_{1}=\frac{9 a_{1} \pi^{2}}{\rho h a^{2}}, \\
& c_{2}=\frac{27 a_{3} \pi^{4}}{4 \rho h a^{4}}, \\
& c_{3}=\frac{81 B A_{2} a_{4} \pi^{6}}{16 \rho b^{4}}, \\
& c_{4}=\frac{729 B A_{2} a_{6} \pi^{10}}{256 \rho h b^{6}}, \\
& f_{1}=\frac{\pi^{2} \bar{N}}{\rho h b^{2}} .
\end{aligned}
$$




\section{Dynamic Characteristics of SMA Composite Wing Subjected to In-Plane Stochastic Excitation}

Equation (12) is a stochastic nonlinear differential equation. The deterministic system corresponding to (12) can be shown as follows:

$$
\ddot{u}_{1}+c_{1} u_{1}+c_{2} u_{1}^{3}+\left(2 \eta_{1}+c_{3} u_{1}^{2}+c_{4} u_{1}^{4}\right) \dot{u}_{1}=0 .
$$
follows:

Let $u_{1}=q$ and $\dot{u}_{1}=\dot{q}=p$; (14) can be rewritten as

$$
\begin{aligned}
& \dot{q}=p \\
& \dot{p}=-2 \eta p-c_{1} q-c_{2} q^{3}-\left(c_{3} q^{2}+c_{4} q^{4}\right) p .
\end{aligned}
$$

The nondamping autonomous system from (15) is shown as follows:

$$
\begin{aligned}
& \dot{q}=p, \\
& \dot{p}=-c_{1} q-c_{2} q^{3} .
\end{aligned}
$$

Its Hamilton function is

$$
H(p, q)=\frac{1}{2} p^{2}+\frac{1}{2} c_{1} q^{2}+\frac{1}{4} c_{2} q^{4} .
$$

According to the quasi-nonintegrable Hamiltonian system theory, the Hamiltonian function $H(t)$ converges weakly in probability to a one-dimensional Ito diffusion process $[29,30]$. The averaged Ito equation about the Hamiltonian function can be shown as follows:

$$
d H=m(H) d t+\sigma(H) d B(t)
$$

where $B(t)$ is standard Wiener process and $m(H)$ and $\sigma(H)$ are the drift and diffusion coefficients of Ito stochastic process, which can be obtained by the stochastic averaging method:

$$
\begin{aligned}
m(H) & =\left(\frac{D e^{2}}{c_{1}}-2 \eta\right) H-\frac{c_{3}}{2 c_{1}} H^{2}-\frac{c_{4}}{2 c_{1}^{2}} H^{3}, \\
\sigma^{2}(H) & =\frac{D e^{2}}{c_{1}} H^{2} .
\end{aligned}
$$

The averaged FPK equation of (18) is

$$
\frac{\partial f}{\partial t}=-\frac{\partial}{\partial H}[m(H) f]+\frac{1}{2} \frac{\partial^{2}\left[\sigma^{2}(H) f\right]}{\partial H^{2}},
$$

where $f=f(H)$ is the stationary probability density (SPD) function of the system's response. Thus,

$$
f(H)=\bar{A} H^{-4 \eta c_{1} / D e^{2}} \exp \left[-\frac{c_{3}}{D e^{2}} H-\frac{c_{4}}{2 D e^{2} c_{1}} H^{2}\right],
$$

where $\bar{A}$ is a normalization constant.

Equation (21) can also be rewritten as follows:

$$
f(H)=H^{-4 \eta c_{1} / D e^{2}} \exp \left[\xi\left(H^{2}+v H+\lambda\right)\right],
$$

where $\xi=-c_{4} / 2 D e^{2} c_{1}, v=2 c_{1} c_{3} / c_{4}$, and $\lambda=\left(-2 D e^{2} c_{1} /\right.$ $\left.c_{4}\right) \ln \bar{A}$. Evidently, $H^{-4 \eta c_{1} / D e^{2}}$ decreases monotonically since $\eta>0, c_{1}>0, D>0$, and $e^{2}>0$. Thus, the bifurcation characteristics of $f(H)$ are only determined by the item $H^{2}+$ $v H+\lambda$.

Now, we can calculate the transition sets of the system response. Let $\bar{G}(H, \lambda, v)=H^{2}+v H+\lambda$ and $H=x^{2}$; we obtain $\bar{G}(x, \lambda, v)=x^{4}+v x^{2}+\lambda$. For $\bar{G}(x, \lambda, v)=x^{4}+v x^{2}+\lambda=0$, we obtain

$$
G(x, \lambda, v)=x \bar{G}(x, \lambda, v)=x^{5}+v x^{3}+\lambda x=0 .
$$

Since $G(-x, \lambda, v)=-G(x, \lambda, v)$, we obtain

$$
g(x, \lambda)=x^{5}+\lambda x=r(\mu, \lambda) x,
$$

where $r(\mu, \lambda)=\mu^{2}+\lambda, \mu=x^{2}$. We have the following:

(1) Bifurcation sets: $G=G_{x}=G_{\lambda}=0$

$$
\begin{aligned}
G & =x^{5}+v x^{3}+\lambda x=0 \\
G_{x} & =5 x^{4}+3 v x^{2}+\lambda=0 \\
G_{\lambda} & =x=0
\end{aligned}
$$

$$
x=0, \lambda=0 .
$$

Thus, the bifurcation set of the system is $(x, \lambda)=$ $(0,0)$.

(2) Hysteretic sets: $G=G_{x}=G_{x x}=0$

$$
\begin{aligned}
& G=x^{5}+v x^{3}+\lambda x=0 \\
& G_{x}=5 x^{4}+3 v x^{2}+\lambda=0 \\
& G_{x x}=20 x^{3}+6 v x=0 \\
& x=0, \lambda=0, x=\sqrt{-\frac{3}{10} v}, \lambda=\frac{21}{100} v^{2} .
\end{aligned}
$$

Thus, the hysteretic sets of the system are $(x, \lambda)=$ $(0,0)$ and $(x, \lambda)=\left(\sqrt{(-3 / 10) v},(21 / 100) v^{2}\right)$, which means $\lambda=(7 / 3) x^{4}$.

(3) Double limited sets: $G=G_{x}=0$

$$
\begin{aligned}
& G=x^{5}+v x^{3}+\lambda x=0 \\
& G_{x}=5 x^{4}+3 v x^{2}+\lambda=0 \\
& x=0, \lambda=0, x=\sqrt{-\frac{1}{2} v}, \lambda=\frac{1}{4} v^{2} .
\end{aligned}
$$

Thus, the double limited sets of the system are $(x, \lambda)=$ $(0,0)$ and $(x, \lambda)=\left(\sqrt{(-1 / 2) v},(1 / 4) v^{2}\right)$, which means $\lambda=x^{4}$.

The transition sets of the system's response are shown in Figure 7. These transfer sets divide the parameter space into three parts, and the corresponding stationary probability 


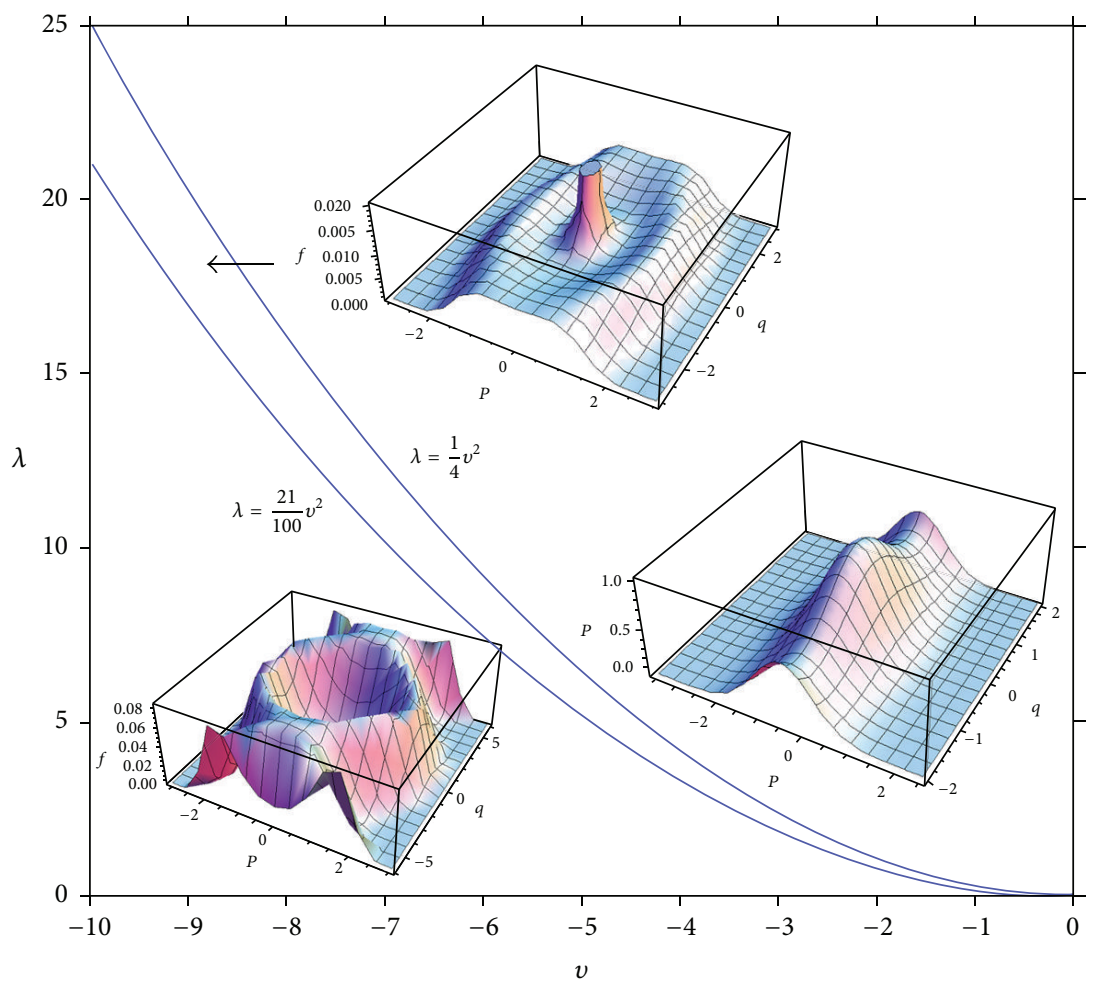

FIGURE 7: Stationary probability density (SPD) of the system response in different parameters.

density functions of the system response in the three parts are also shown in Figure 6, where $c_{1}=2500, c_{2}=-100, c_{3}=20$, $D=0.5, \eta=0.02$, and $l=1$.

From Figure 7, we can see that

(1) when $\lambda>(1 / 4) v^{2}$, the steady-state probability density of $H=0$ is the maximum, and there is a crest in the SPD of the system response;

(2) when $(21 / 100) v^{2}<\lambda<(1 / 4) v^{2}$, there is a crest and a loop in the SPD of the system response; although the steady-state probability density of $H=0$ is also the maximum, it decreases with the variation of the parameters;

(3) when $\lambda<(21 / 100) v^{2}$, there is a loop in the SPD of the system response, and the loop has the maximum SPD; it means that the system's motion is the stochastic vibration near a periodic orbit;

(4) in sum, stochastic Hopf bifurcation appears in the variation of the parameters.

\section{Optimal Control to SMA Composite Wing}

According to the results of the above section, the stochastic Hopf bifurcation appears in the process of varying the system parameters. Bifurcation changes the system's motion and decreases its reliability. In this section, stochastic optimal control is introduced to improve the system's reliability.
The dynamic model of controlled system can be shown as follows:

$$
\begin{aligned}
\dot{q}= & p, \\
\dot{p}= & -2 \eta p-c_{1} q-c_{2} q^{3}-\left(c_{3} q^{2}+c_{4} q^{4}\right) p+e q \xi(t) \\
& +F(t),
\end{aligned}
$$

where $F(t)$ is the active control force. To SMA beam, the active control force is supplied by heating and cooling. The Ito diffusion process of (28) can be shown as follows:

$$
\begin{aligned}
d H= & {\left[m(H)+\frac{1}{T(H)} \int_{\Gamma} F(t) d q\right] d t } \\
& +\sigma(H) d B(t),
\end{aligned}
$$

where $T(H)=\int_{\Gamma}(1 / p) d q, \Gamma=\left\{q \mid(1 / 2) k q^{2} \leq H\right\}$.

The reliability function $R(H, t)$ of the system is determined by the dynamic programming principle shown as follows:

$$
\begin{aligned}
\frac{\partial R}{\partial t} & =-\inf _{F}\left\{x^{T} A^{T} \frac{\partial R}{\partial x}+F^{T} \bar{D}^{T} \frac{\partial R}{\partial x}\right. \\
& \left.+\frac{1}{2}\left(\sigma \sigma^{T}\right)_{i j} \frac{\partial^{2} R}{\partial x_{i} \partial x_{j}}+x^{T} Q x+F^{T} R F\right\} .
\end{aligned}
$$

The optimal control force $F^{*}$ should make the reliability function the maximum, so it can be determined as follows:

$$
F^{*}=-\frac{1}{2} R^{-1} \bar{D}^{T} \frac{\partial R}{\partial x} .
$$


The stress $\sigma=F^{*}(t) / \bar{A}$ can be obtained when the control force $F^{*}$ is determined, where $\sigma$ is the stress and $\bar{A}$ is the area of the cross section of SMA plate. The vertical displacement can be measured by sensors, and the strain of SMA plate $\varepsilon$ can be calculated. Thus, the input temperature $T$ can be determined according to Auricchio's SMA constitutive model as follows [22]:

$$
\sigma=E \varepsilon^{e}=E\left[\varepsilon-\xi_{S} \beta+\xi_{R}(\beta-k)-\alpha\left(T-T_{0}\right)\right],
$$

where $E$ is the elasticity modulus, $\xi_{\mathrm{S}} \beta$ is the inelastic strain induced by phase change, and $\alpha$ is the thermal expansion coefficient. The input current intensity $I$ can be determined as follows:

$$
\dot{T}=e_{1} I^{2} R-e_{2}\left(T-T_{0}\right),
$$

where $e_{1}$ and $e_{2}$ are the heat transfer coefficients.

Substituting (31) into (29), we obtained

$$
d H=\left[m(H)-\frac{2 \sqrt{2} b}{\pi} H^{1 / 2}\right] d t+\sigma(H) d B(t) .
$$

Thus, the background Kolmogorov equation (BK equation) can be shown as follows:

$$
\frac{\partial R}{\partial t}=\left[m(H)-\frac{2 \sqrt{2} b}{\pi} H^{1 / 2}\right] \frac{\partial R}{\partial H}+\frac{1}{2} \sigma^{2}(H) \frac{\partial^{2} R}{\partial H^{2}} .
$$

The initial condition is

$$
R\left(H_{0}, 0\right)=1, \quad H_{0} \in \Gamma \text { when } t=0 .
$$

The boundary conditions are

$$
\begin{aligned}
R\left(H_{0}, t\right) & =0 \quad \text { when } H_{0}=\Gamma \\
\frac{\partial R}{\partial t} & =\left[m(H)-\frac{2 \sqrt{2} b}{\pi} H^{1 / 2}\right] \frac{\partial R}{\partial H}
\end{aligned}
$$

when $H_{0}=0$.

The probability density $p\left(H_{0}, t\right)$ of the first-passage time (FPT) of the controlled system is determined as follows:

$$
p\left(H_{0}, T\right)=-\left.\frac{\partial R}{\partial t}\right|_{t=T} .
$$

The numerical simulation results of the controlled system reliability function are shown in Figure 8, and the probability density of first-passage time is shown in Figure 9, where $D=$ $1, H=3$, and $b=0 / 1 / 3$, where $b$ is the mean intensity of control force. In Figure 8, the abscissa is time $(t)$, whose unit is second (s); the ordinate is the reliability of the optimal controlled system $\left(R_{\text {opt }}\right)$, whose unit is $100 \%$. In Figure 9, the abscissa is the first-passage time $(T)$, whose unit is second $(\mathrm{s})$; the ordinate is the probability density of the system's firstpassage time $\left(P_{\text {opt }}\right)$, whose unit is $100 \%$.

From Figures 8 and 9, we can see the following:

(1) The reliability function $R(H, t)$ decreases as time increases.

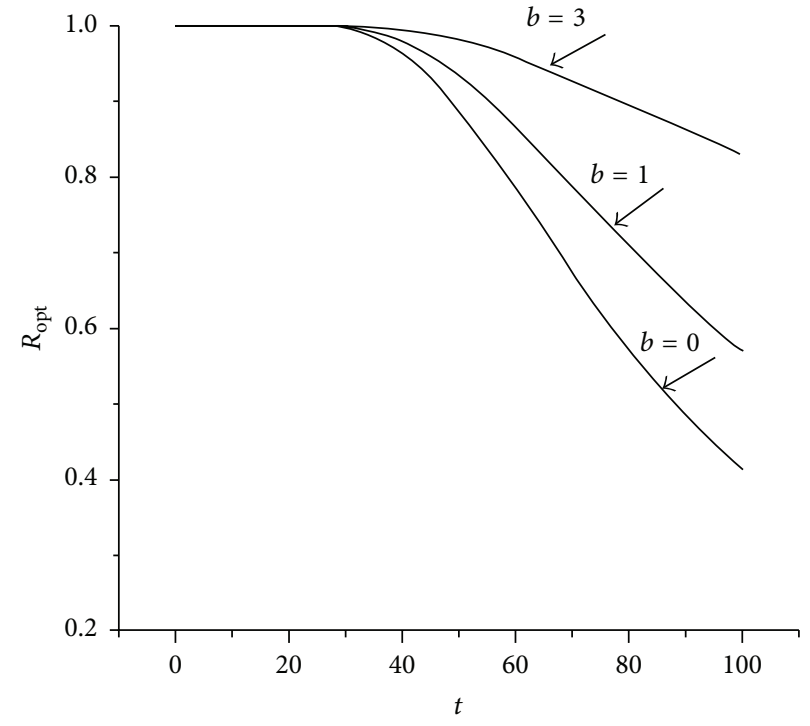

Figure 8: Reliability function of the system.

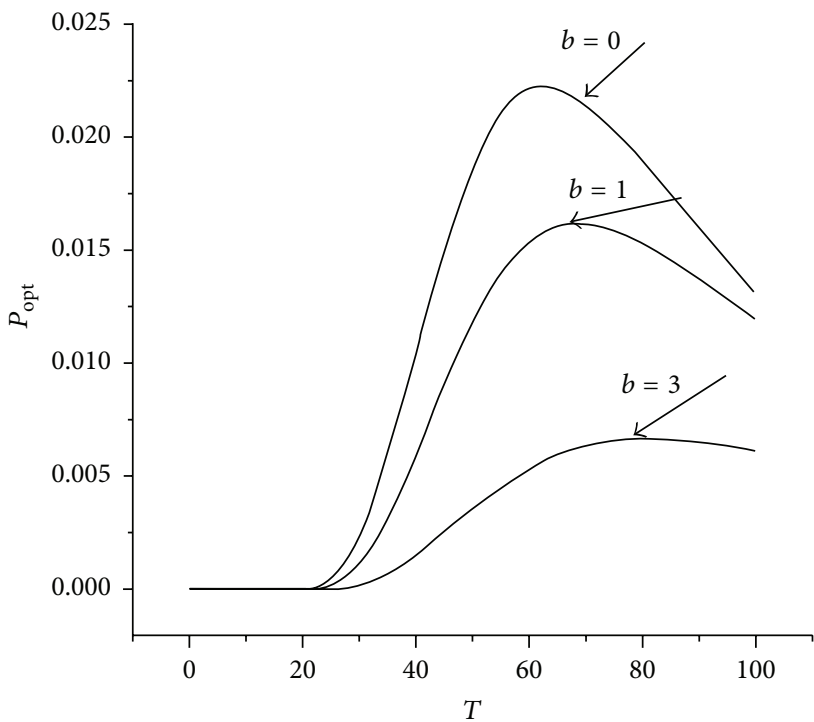

FIGURE 9: Probability density of first-passage time.

(2) The system's reliability is obviously improved when the control force increases, which means that the control strategy is effective.

(3) The probability density of first-passage time increases with time. First passage means the system leaves the safe area, which causes the system's instability.

(4) First-passage time can be delayed through optimal control, which also means that the optimal control can enhance the system's reliability.

\section{Experiments}

The experimental object is a 1:26 Chinese "Wing Loong" UAV model. The model is shown in Figure 10, and its parameters 
TABLE 1: Model's parameters.

\begin{tabular}{llcc}
\hline Wing's mass $(\mathrm{g})$ & 12.8 & SMA plate's mass $(\mathrm{g})$ & 2.68 \\
Wing's length $(\mathrm{cm})$ & 19.4 & SMA plate's length $(\mathrm{cm})$ & 12 \\
Wing's width $(\mathrm{cm})$ & 1.24 & SMA plate's width $(\mathrm{cm})$ & 0.7 \\
Wing's thickness $(\mathrm{mm})$ & 1.97 & SMA plate's thickness $(\mathrm{mm})$ & 0.4 \\
\hline
\end{tabular}

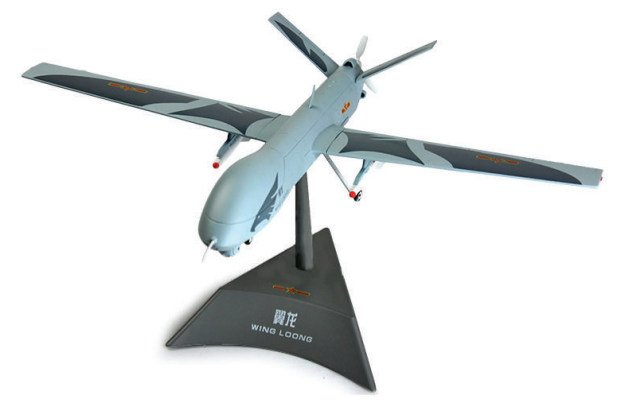

FIgURE 10: 1:14 Chinese "Wing Loong” UAV model.

are shown in Table 1. All the data in Table 1 are measured by us. The model is supplied by Hongda Model Company, Beijing, China. SMA thin plate is pasted on the inner surface of the wing. The SMA thin plate is made up of several SMA thin strips. The SMA thin strips are supplied by Tianxin Company, Gansu Province, China. The SMA's hysteresis is shown in Figure 1. Its transient temperatures are $\mathrm{Ms}=243.4 \mathrm{~K}$, $\mathrm{Mf}=223.2 \mathrm{~K}, \mathrm{As}=304.7 \mathrm{~K}$, and $\mathrm{Af}=311.7 \mathrm{~K}$. The system is in a low-speed wind tunnel. The stochastic airflow is produced by adding random guide vanes in the low-speed wind tunnel. The heating method for SMA plate is by heating wire, and the cooling is natural. The vibration amplitude of the wingtip is measured by vibration sensors. The control process is as follows:

(1) Firstly, we measure the vibration of the wingtip by vibration sensors and obtained the displacement $q$ and the velocity $p$, and then we can calculate the strain $\varepsilon$.

(2) Secondly, we calculate $\partial R / \partial t$ according to (30) and then obtain $R(t)$ through integration.

(3) Thirdly, we calculate the optimal control force $F^{*}$ according to (31).

(4) When the optimal control force $F^{*}$ is given, we can obtain the control stress $\sigma=F^{*}(t) / \bar{A}$.

(5) When the control stress $\sigma$ and the strain $\varepsilon$ are both determined, we can calculate the control temperature $T$ according to (32).

(6) Finally, we obtained the intensity of control current $I$ according to (33).

The experimental results of the SMA composite wing subjected to stochastic airflow are shown in Figures 11-20.

From the experimental results, we can see that

(1) the system's motion is the mixture of periodic motion and stochastic motion;

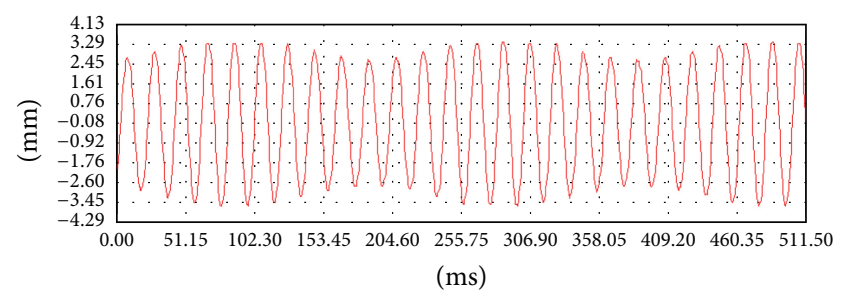

FIGURE 11: Wingtip's dynamic response of an uncontrolled SMA composite wing when the mean wind velocity $V=6 \mathrm{~m} / \mathrm{s}$.

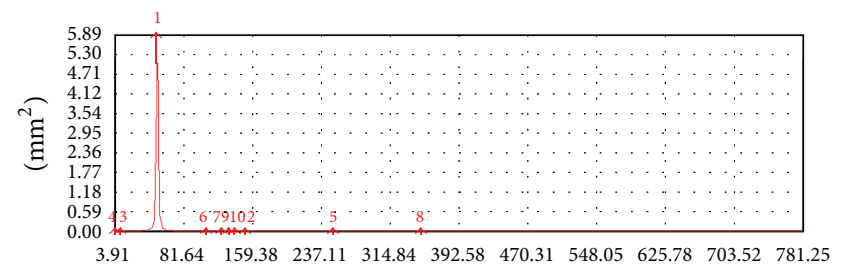

$(\mathrm{Hz})$

FIGURE 12: Frequency domain of the wingtip's dynamic response of an uncontrolled SMA composite wing when the mean wind velocity $V=6 \mathrm{~m} / \mathrm{s}$.

(2) the system's vibration increases with the mean wind velocity; when the wind velocity increases from $6 \mathrm{~m} / \mathrm{s}$ to $8 \mathrm{~m} / \mathrm{s}$, the vibration amplitude of wingtip increases from $3.32 \mathrm{~mm}$ to $4.21 \mathrm{~mm}$, which rises by $26 \%$;

(3) the vibration amplitude of an uncontrolled wingtip is $4.21 \mathrm{~mm}$ when the mean wind velocity $V=8 \mathrm{~m} / \mathrm{s}$, and the one of a controlled wingtip is only $3.48 \mathrm{~mm}$; it means that the vibration amplitude of the wingtip is reduced by $17.4 \%$ under the control; in fact, the control effect is not very evident; we think the reason is that the time-delayed characteristics of SMA are not considered in the control process, which cause the SMA actuator's action being not very accurate; if the response time of SMA is considered and the control strategy is developed accordingly, SMA composite beam is a promising way to control the wing's fluttering.

\section{Conclusions}

In this paper, a kind of high-aspect-ratio shape memory alloy (SMA) composite wing is proposed to reduce the wing's fluttering. The nonlinear dynamic characteristics and optimal control of the SMA composite wings subjected to inplane stochastic excitation are investigated where the great bending under the flight loads is considered. The stochastic stability of the system is analyzed, and the system's response is obtained. The conditions of stochastic Hopf bifurcation are determined, and the probability density of the first-passage time is obtained. Finally, the optimal control strategy is proposed. Numerical simulation shows that the stability of the system varies with bifurcation parameters, and stochastic Hopf bifurcation occurs in the process; the reliability of 


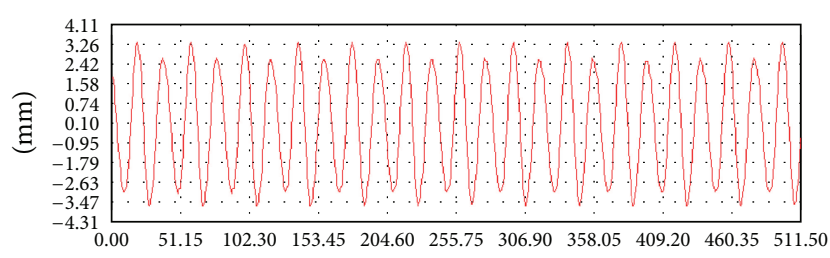

(ms)

FIGURE 13: Wingtip's dynamic response of a controlled SMA composite wing when the mean wind velocity $V=6 \mathrm{~m} / \mathrm{s}$.

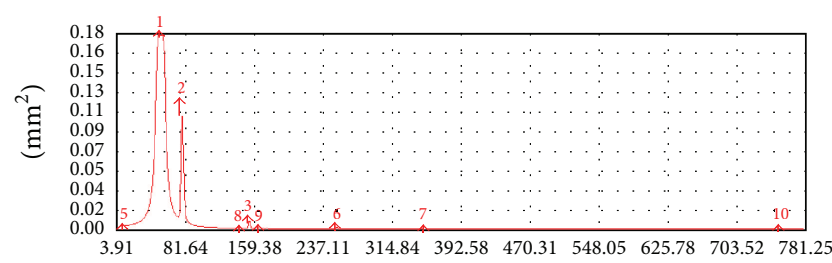

$(\mathrm{Hz})$

FIGURE 14: Frequency domain of the wingtip's dynamic response of a controlled SMA composite wing when the mean wind velocity $V=$ $6 \mathrm{~m} / \mathrm{s}$.

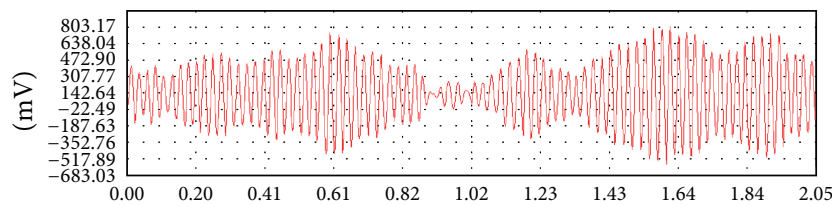

(s)

FIGURE 15: Voltage of the control current when the mean wind velocity $V=6 \mathrm{~m} / \mathrm{s}$.

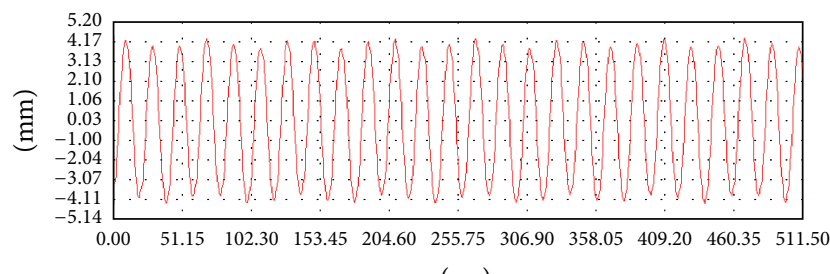

(ms)

FIGURE 16: Wingtip's dynamic response of an uncontrolled SMA composite wing when the mean wind velocity $V=8 \mathrm{~m} / \mathrm{s}$.

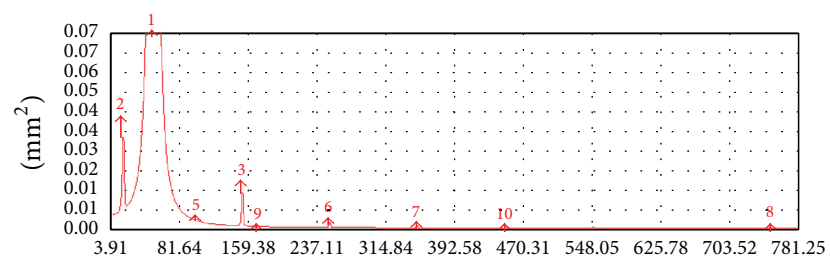

$(\mathrm{Hz})$

FIGURE 17: Frequency domain of the wingtip's dynamic response of an uncontrolled SMA composite wing when the mean wind velocity $V=8 \mathrm{~m} / \mathrm{s}$.

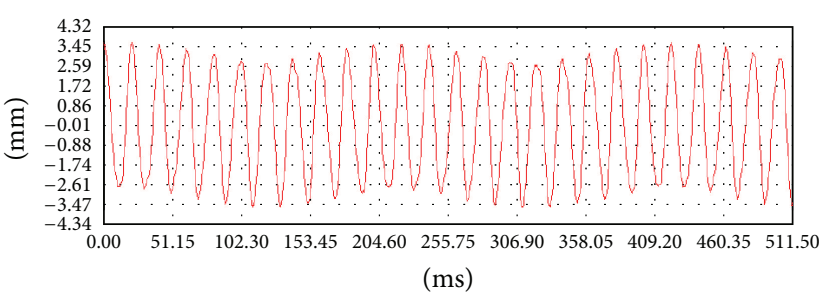

FIGURE 18: Wingtip's dynamic response of a controlled SMA composite wing when the mean wind velocity $V=8 \mathrm{~m} / \mathrm{s}$.

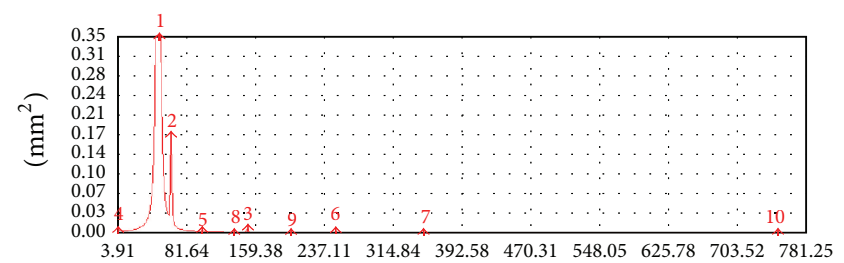

$(\mathrm{Hz})$

FIGURE 19: Frequency domain of the wingtip's dynamic response of a controlled SMA composite wing when the mean wind velocity $V=$ $8 \mathrm{~m} / \mathrm{s}$.

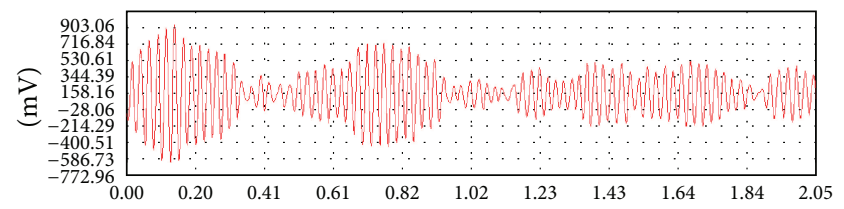

(s)

FIGURE 20: Voltage of the control current when the mean wind velocity $V=8 \mathrm{~m} / \mathrm{s}$.

the system is improved through optimal control, and the firstpassage time is delayed. Finally, the effects of the control strategy are proved by experiments. The results of this paper are helpful for engineering applications of SMA.

\section{Conflict of Interests}

The authors declare that there is no conflict of interests regarding the publication of this paper.

\section{Acknowledgments}

The authors gratefully acknowledge the support of the Natural Science Foundation of China (NSFC) through Grants nos. 11272229, 11302144, and 11402168, the Ph.D. Programs Foundation of the Ministry of Education of China through Grant no. 20120032120006, and the Tianjin Research Program of Application Foundation and Advanced Technology through Grants nos. 13JCYBJC17900 and 14JCQNJC05300.

\section{References}

[1] S.-M. Yang, J.-H. Han, and I. Lee, "Characteristics of smart composite wing with SMA actuators and optical fiber sensors," 
International Journal of Applied Electromagnetics and Mechanics, vol. 23, no. 3, pp. 177-186, 2006.

[2] G. Mirone, "Design and demonstrators testing of adaptive airfoils and hingeless wings actuated by shape memory alloy wires," Smart Structures and Systems, vol. 3, no. 1, pp. 89-114, 2007.

[3] U. Icardi and L. Ferrero, "Preliminary study of an adaptive wing with shape memory alloy torsion actuators," Materials and Design, vol. 30, no. 10, pp. 4200-4210, 2009.

[4] U. Icardi and L. Ferrero, "SMA actuated mechanism for an adaptive wing," Journal of Aerospace Engineering, vol. 24, no. 1, pp. 140-143, 2011.

[5] S. Barbarino, S. Ameduri, L. Lecce, and A. Concilio, "Wing shape control through an SMA-based device," Journal of Intelligent Material Systems and Structures, vol. 20, no. 3, pp. 283-296, 2009.

[6] T. L. Grigorie and R. M. Botez, "New adaptive controller method for SMA hysteresis modelling of a morphing wing," Aeronautical Journal, vol. 114, no. 1151, pp. 1-13, 2010.

[7] S. H. Kim and M. H. Cho, "A simple smart wing actuator using Ni-Ti SMA," Journal of Mechanical Science and Technology, vol. 24, no. 9, pp. 1865-1873, 2010.

[8] M. M. Barzegari, M. Dardel, A. Fathi, and M. Ghadimi, "Aeroelastic characteristics of cantilever wing with embedded shape memory alloys," Acta Astronautica, vol. 79, no. 10, pp. 189202, 2012.

[9] W.-R. Kang, E.-H. Kim, M.-S. Jeong, I. Lee, and S.-M. Ahn, "Morphing wing mechanism using an SMA wire actuator," International Journal of Aeronautical and Space Sciences, vol. 13, no. 1, pp. 58-63, 2012.

[10] J. Colorado, A. Barrientos, and C. Rossi, "Biomechanics of smart wings in a bat robot: morphing wings using SMA actuators," Bioinspiration and Biomimetics, vol. 7, no. 3, Article ID 036006, 10 pages, 2012.

[11] K.-T. Lau, "Vibration characteristics of SMA composite beams with different boundary conditions," Materials and Design, vol. 23, no. 8, pp. 741-749, 2002.

[12] K. M. Liew, J. Ren, and S. Kitipornchai, "Analysis of the pseudoelastic behavior of a SMA beam by the element-free Galerkin method," Engineering Analysis with Boundary Elements, vol. 28, no. 5, pp. 497-507, 2004.

[13] A. Zbiciak, "Dynamic analysis of pseudoelastic SMA beam," International Journal of Mechanical Sciences, vol. 52, no. 1, pp. 56-64, 2010.

[14] F. Scarpa, M. Ruzzene, and M. R. Hassan, "Spectral element formulation for SMA beams under random vibration excitation," Smart Materials and Structures, vol. 33, no. 8, pp. 286-293, 2004.

[15] S. M. T. Hashemi and S. E. Khadem, "Modeling and analysis of the vibration behavior of a shape memory alloy beam," International Journal of Mechanical Sciences, vol. 48, no. 1, pp. 44-52, 2006.

[16] M. Collet, E. Foltête, and C. Lexcellent, "Analysis of the behavior of a shape memory alloy beam under dynamical loading," European Journal of Mechanics A/Solids, vol. 20, no. 4, pp. 615630, 2001.

[17] K. Tanaka, "A thermomechanical sketch of shape memory effect: one-dimensional tensile behavior," Res Mechanica, vol. 18, no. 2, pp. 251-263, 1986.

[18] J. G. Boyd and D. C. Lagoudas, "A thermodynamical constitutive model for shape memory materials. Part I. The monolithic shape memory alloy," International Journal of Plasticity, vol. 12, no. 6, pp. 805-842, 1996.
[19] L. C. Brinson, "One-dimensional constitutive behavior of shape memory alloys: thermomechanical derivation with nonconstant material functions and redefined martensite internal variable," Journal of Intelligent Material Systems and Structures, vol. 4, no. 2, pp. 229-242, 1993.

[20] E. J. Graesser and F. A. Cozzarelli, "Proposed three-dimensional constitutive model for shape memory alloys," Journal of Intelligent Material Systems and Structures, vol. 5, no. 1, pp. 78-89, 1994.

[21] Y. Ivshin and T. J. Pence, "Thermomechanical model for a one variant shape memory material," Journal of Intelligent Material Systems and Structures, vol. 5, no. 4, pp. 455-473, 1994.

[22] F. Auricchio and J. Lubliner, "Uniaxial model for shape-memory alloys," International Journal of Solids and Structures, vol. 34, no. 27, pp. 3601-3618, 1997.

[23] M. A. Savi, P. M. C. L. Pacheco, and A. M. B. Braga, "Chaos in a shape memory two-bar truss," International Journal of NonLinear Mechanics, vol. 37, no. 8, pp. 1387-1392, 2002.

[24] J. W. Sohn, Y. M. Han, S. B. Choi, Y. S. Lee, and M. S. Han, "Vibration and position tracking control of a flexible beam using SMA wire actuators," Journal of Vibration and Control, vol. 15, no. 2, pp. 263-281, 2009.

[25] H. Li, Z.-Q. Liu, and J.-P. Ou, "Experimental study of a simple reinforced concrete beam temporarily strengthened by SMA wires followed by permanent strengthening with CFRP plates," Engineering Structures, vol. 30, no. 3, pp. 716-723, 2008.

[26] C. G. Wang and H. F. Tan, "Experimental and numerical studies on wrinkling control of an inflated beam using SMA wires," Smart Materials and Structures, vol. 19, no. 10, Article ID 105019, pp. 1025-1031, 2010.

[27] M. S. Speicher, R. DesRoches, and R. T. Leon, "Experimental results of a NiTi shape memory alloy (SMA)-based recentering beam-column connection," Engineering Structures, vol. 33, no. 9, pp. 2448-2457, 2011.

[28] Z.-W. Zhu, W.-Y. Xie, and J. Xu, "Nonlinear dynamic characteristics of SMA simply supported beam in axial stochastic excitation," Journal of Vibroengineering, vol. 14, no. 3, pp. 10591067, 2012.

[29] W. Q. Zhu and Z. L. Huang, "Stochastic stability of quasinon-integrable-Hamiltonian systems," Journal of Sound and Vibration, vol. 218, no. 5, pp. 769-789, 1998.

[30] W. Q. Zhu and Z. L. Huang, "Stochastic Hopf bifurcation of quasi-nonintegrable-Hamiltonian systems," International Journal of Non-Linear Mechanics, vol. 34, no. 3, pp. 437-447, 1999. 

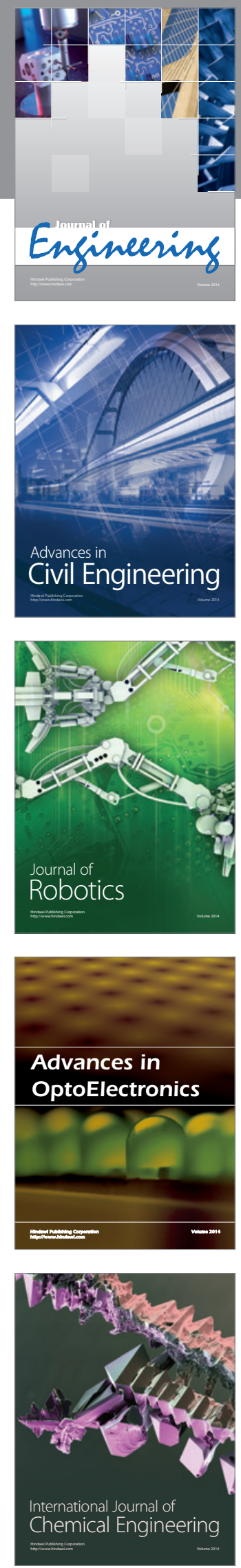

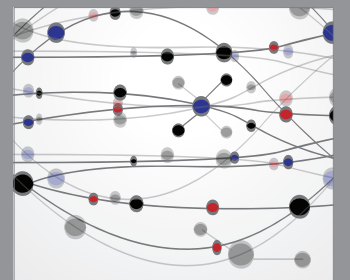

The Scientific World Journal
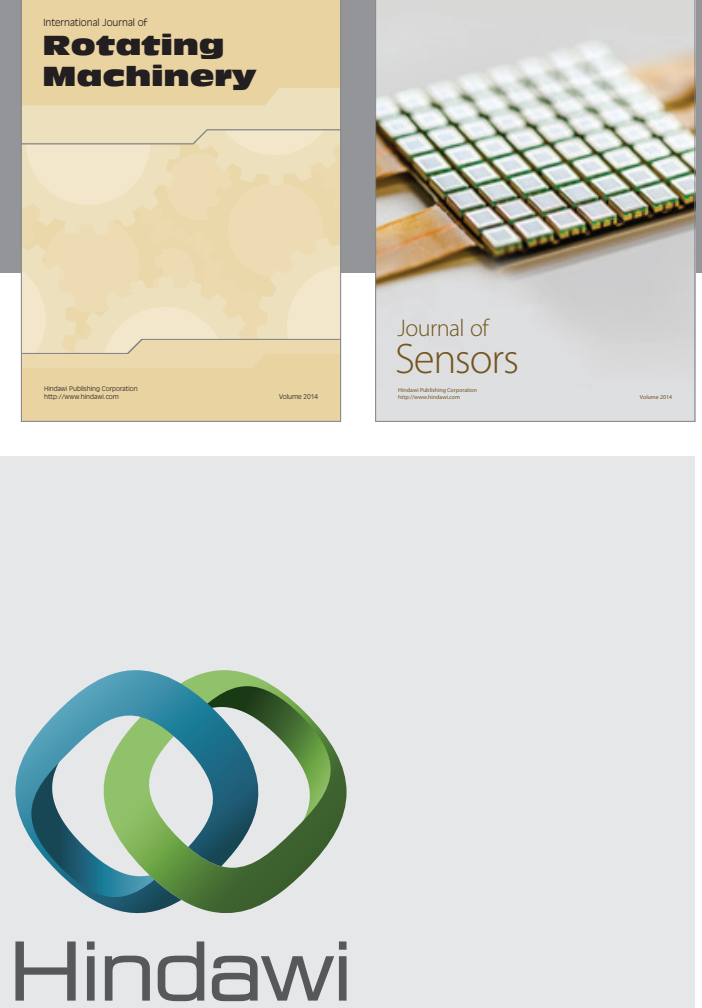

Submit your manuscripts at http://www.hindawi.com
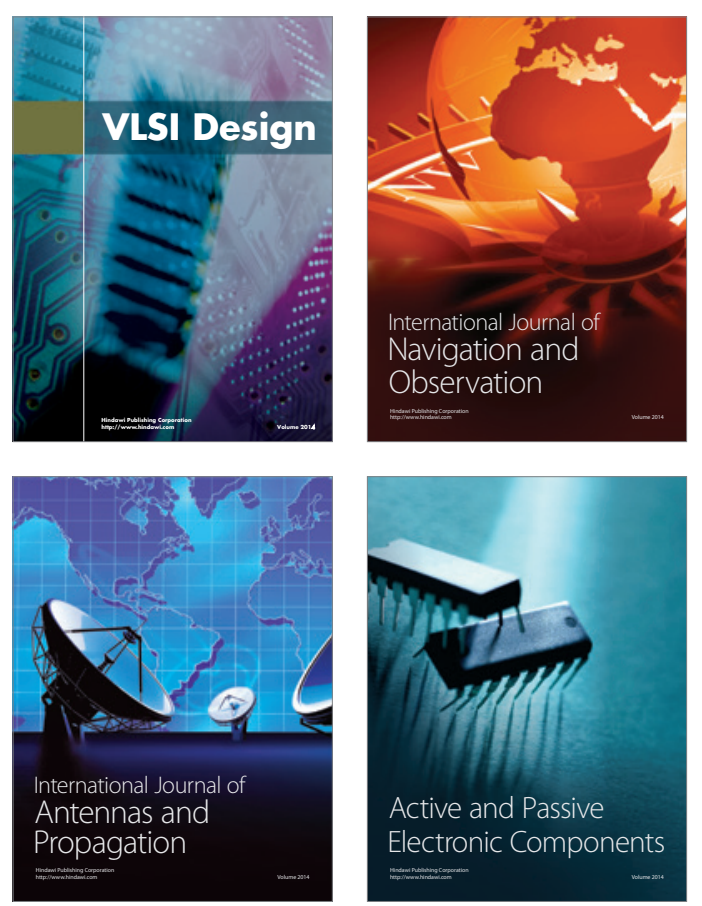
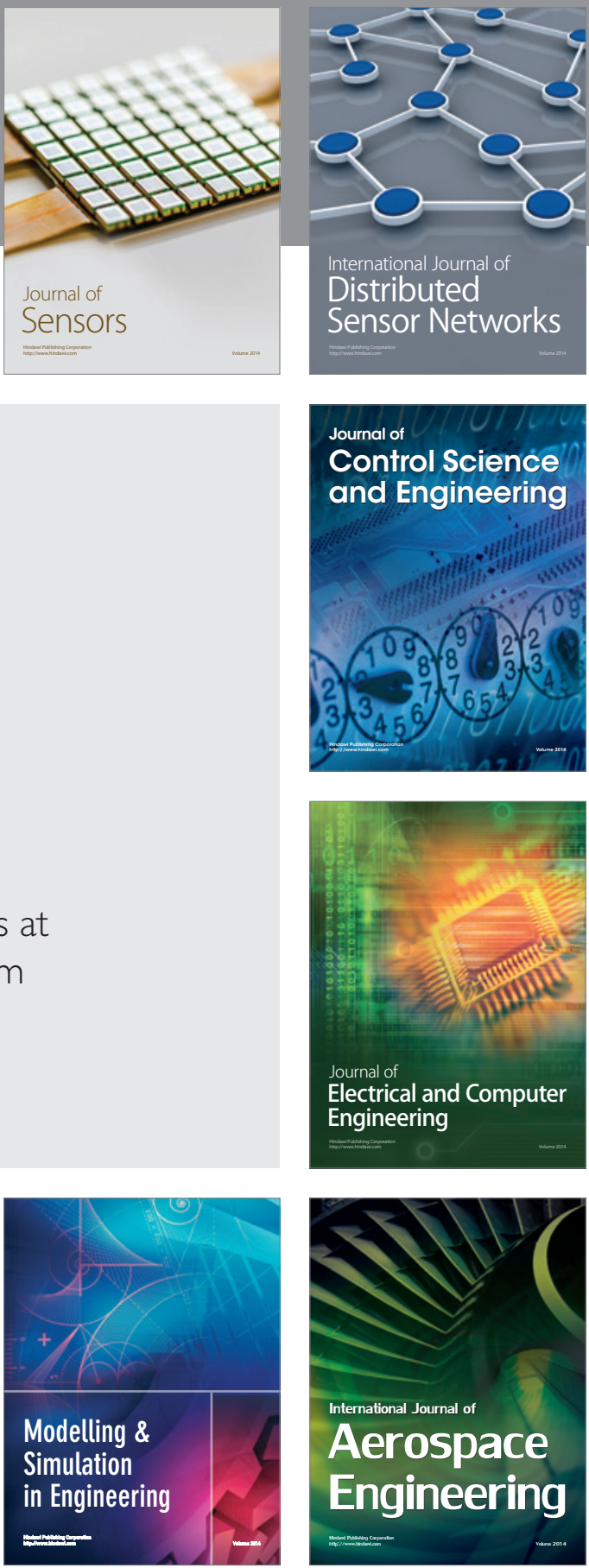

Journal of

Control Science

and Engineering
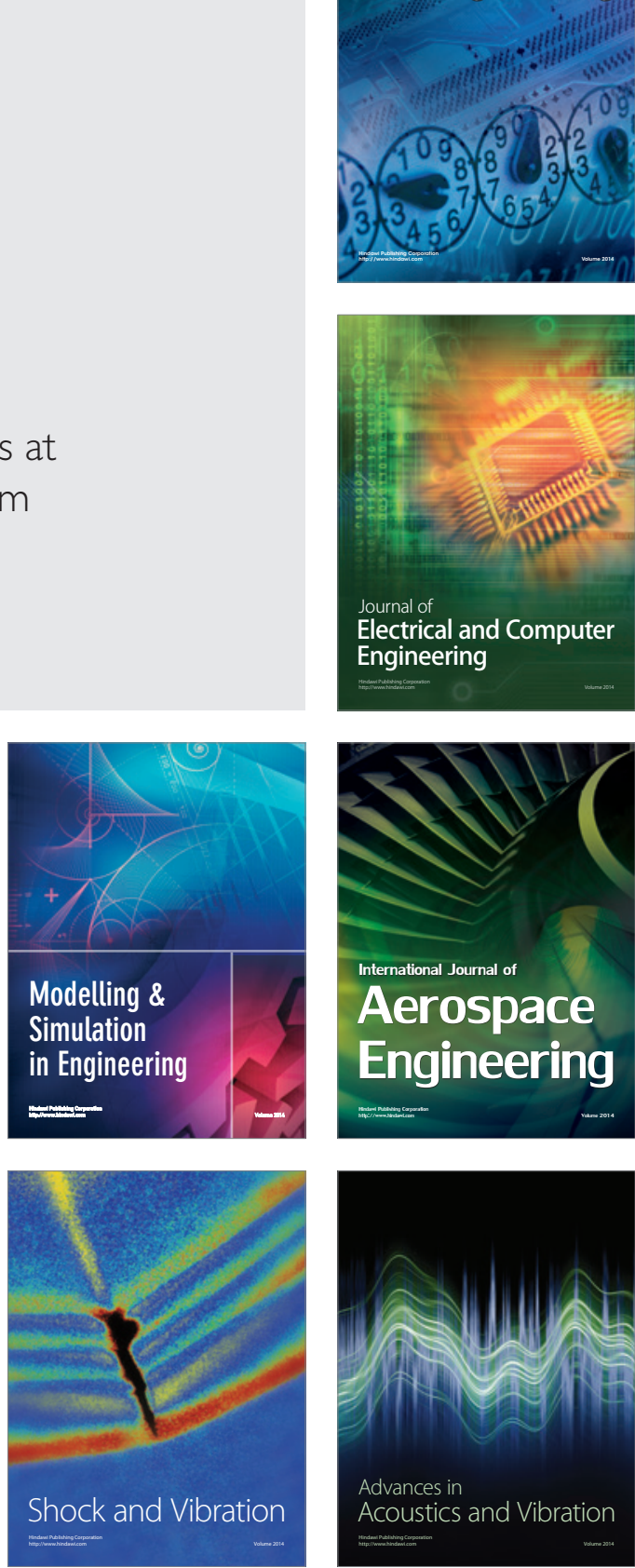